\title{
Solubility of Clonazepam, Diazepam, Lamotrigine, and Phenobarbital in $N$-Methyl-2-pyrrolidone + Water Mixtures at 298.2 K
}

\author{
Ali Shayanfar, ${ }^{\dagger}$ William E. Acree, Jr., ${ }^{\dagger}$ and Abolghasem Jouyban ${ }^{*}$, \\ Biotechnology Research Center, Tabriz University (Medical Sciences), Tabriz 51664, Iran, Faculty of Pharmacy and Drug \\ Applied Research Center, Tabriz University (Medical Sciences), Tabriz 51664, Iran, and Department of Chemistry, \\ University of North Texas, Denton, Texas 76203-5070
}

\begin{abstract}
Experimental solubilities of clonazepam, diazepam, lamotrigine, and phenobarbital in binary solvent mixtures of $N$-methyl-2-pyrrolidone (NMP) + water at $298.2 \mathrm{~K}$ were reported. The solubility of clonazepam, diazepam, and lamotrigine was increased with the addition of NMP, and maximum values are in neat NMP. The solubility of phenobarbital was increased with addition of NMP, reached the maximum value, and then decreased with further increase in NMP concentration. The Jouyban-Acree model was fitted to the results of these measurements, and solubilities were back-calculated by employing the solubility data in monosolvents in which the overall mean deviation of the models was $10.7 \%$.
\end{abstract}

\section{Introduction}

Solubility is an important physicochemical parameter in pharmaceutical sciences. The aqueous solubility of drugs is one of the important key parameters to make decisions on the fate of a drug candidate. In drug development, solubility data are essential information for the preparation of drug formulations, solid phase properties, and correlation between in vitro/in vivo data. Aqueous solubility is also required to make a solution of the drug for pharmacological and toxicological tests of drug candidates. Solubility of drugs in nonaqueous solvent is important in synthesis of drugs and recrystallization for the purification process and development of pharmaceutical analysis. ${ }^{1}$

Solubilization of poorly soluble drugs is essential for the preparation of many commercially available oral solution, parenteral, soft gelatin, and topical pharmaceutical formulations of drugs. ${ }^{2}$ A number of methods have been developed for solubilization of drugs including the cosolvency method. Cosolvency is mixing of a water-soluble organic solvent that is miscible with water for decreasing the polarity of dissolution medium. The main advantages of the cosolvency method are its solubilization power and ease of use. ${ }^{3}$ The prediction of physicochemical properties in pharmaceutical sciences is very important. About cosolvency, efforts have been devoted to the presentation of mathematical models for estimation of drug solubility in water-cosolvent mixtures. These models ${ }^{4-12}$ were recently reviewed, and their advantages and limitations were discussed. ${ }^{13}$ One of these models developed by our group is the Jouyban-Acree model. This model was used for prediction of the solubility of many pharmaceutical and chemical compounds in binary, ternary, and quaternary solvent mixtures at different temperatures. In addition to the solubility prediction, it was used to calculate other physicochemical properties in mixed solvent systems. ${ }^{13}$

\footnotetext{
* Author to whom correspondence should be addressed. E-mail: ajouyban@ hotmail.com. Fax: +98 4113363231 .

† Biotechnology Research Center.

$\because$ University of North Texas.

${ }^{\S}$ Faculty of Pharmacy and Drug Applied Research Center.
}

Common cosolvents in pharmacy are ethanol, propylene glycol, glycerin, polyethylene glycol 400, and N-methyl-2pyrrolidone (NMP). ${ }^{14} \mathrm{NMP}$ is a very strong solubilizing agent ${ }^{2}$ and is an important solvent in extraction, purification, and crystallization of drugs. ${ }^{15,16}$ Solubility data of cefotaxime, ${ }^{17}$ dioxopromethazine hydrochloride, ${ }^{18}$ and benzoic acid ${ }^{19}$ in NMP at different temperatures and in NMP + water, up to $50 \%$ of NMP for estrone and griseofulvin, ${ }^{20}$ have been reported. Experimental solubilities of some antiepileptic drugs in ethanol + water and propylene glycol + water mixtures were reported in previous works. ${ }^{21,22}$ However, there are no published data of the investigated drugs in the literature. Hence, in this work, the solubilities of clonazepam (CZP), diazepam (DZP), lamotrigine (LTG), and phenobarbital (PB) in NMP + water mixtures at $298.2 \mathrm{~K}$ were reported, and the fitness of the data to the Jouyban-Acree model was investigated.

\section{Experimental Methods}

DZP (99.7 \% in mass fraction) and CZP (99.7 \% in mass fraction) were gifts from Sobhan pharmaceutical (Iran). PB (99.8 $\%$ in mass fraction) was a gift from Pars Daru pharmaceutical (Iran), and LTG (99.6\% in mass fraction) was purchased from Arastoo pharmaceutical (Iran). The purity of the drugs was checked by determination of their melting temperatures and comparing the measured solubilities in solvents with the reported data from the literature. NMP (99.5\% in mass fraction) from Merck (Germany) and methanol ( $99.8 \%$ in mass fraction) from Caledon (Canada) were purchased, and double distillated water (with the electrical conductivity of $<3 \cdot 10^{-6} \mathrm{~S}$ ) was used for preparation of the solutions.

\section{Apparatus and Procedures}

The binary mixtures composed of NMP + water with suitable volumes of the solvents were prepared with the uncertainty of 0.001 volume fraction. The solubility data of CZP, DZP, LTG, and $\mathrm{PB}$ in NMP + water were determined by the saturation shake-flask method. ${ }^{23}$ In this method, an excess amount of the drugs was added to the prepared solutions, then these solutions were saturated in an incubator equipped with a temperature- 
Table 1. Details of Calibration Curves of Drugs

\begin{tabular}{|c|c|c|}
\hline & $\varepsilon$ & $C$ \\
\hline drug & $\left(\mathrm{L} \cdot \mathrm{mol}^{-1} \cdot \mathrm{cm}^{-1}\right)$ & $\left(\mathrm{mol} \cdot \mathrm{L}^{-1}\right)$ \\
\hline LTG & 6681 to 6904 & $3.59 \cdot 10^{-5}$ to $1.80 \cdot 10^{-4}$ \\
\hline DZP & 45217 to 77215 & $3.48 \cdot 10^{-6}$ to $2.79 \cdot 10^{-5}$ \\
\hline $\mathrm{CZP}$ & 11297 to 12050 & $1.66 \cdot 10^{-5}$ to $1.33 \cdot 10^{-4}$ \\
\hline PB & 3753 to 3073 & $8.53 \cdot 10^{-5}$ to $4.26 \cdot 10^{-4}$ \\
\hline
\end{tabular}

controlling system maintained constant at $298.2( \pm 0.2) \mathrm{K}$ and using a shaker (Behdad, Tehran, Iran). Also for PB powders in a binary solvent mixture before incubation at $298.2 \mathrm{~K}$, solutions were sonicated for $20 \mathrm{~min}$. After a sufficient length of time (> 5 days for $\mathrm{PB}$ and $>3$ days for other drugs), the saturated solutions of the drugs were filtered using hydrophilic Durapore filters $(0.45 \mu \mathrm{m}$, Milipore, Ireland) diluted with water for LTG and with methanol for CZP, DZP, and PB. Diluted samples were then assayed at $(306,229,309$, and 230) nm, respectively, using a UV-vis spectrophotometer (Beckman DU-650, Fullerton, USA). Preliminary investigations showed that the filter did not absorb the solutes through the filtration process. The concentrations of solutions were determined according to the calibration curves. Details of calibration curves were shown in Table 1. Each experimental data point represents the average of at least three repeated measurements with the measured mole per liter solubilities being reproducible to within $\pm 4.1 \%$. Calculated standard deviations ranged from $\left(\sigma_{n-1}=0.0000029\right.$ to $\sigma_{n-1}=$ $0.1455292) \mathrm{mol} \cdot \mathrm{L}^{-1}$. Densities of the saturated solutions were determined using a $5 \mathrm{~mL}$ pycnometer with the uncertainty of $0.0001 \mathrm{~g} \cdot \mathrm{cm}^{-3}$.

\section{Computational Methods}

The general form of the Jouyban-Acree model for calculation of solubility in binary solvent mixtures at different temperatures is $^{13}$

$$
\begin{aligned}
\log C_{\mathrm{m}, \mathrm{T}}^{\mathrm{Sat}}=\varphi_{1} \log C_{1, \mathrm{~T}}^{\mathrm{Sat}}+\varphi_{2} \log C_{2, \mathrm{~T}}^{\mathrm{Sat}}+ & \\
& {\left[\frac{\varphi_{1} \varphi_{2}}{T} \sum_{i=0}^{2} J_{i}\left(x_{1}-x_{2}\right)^{i}\right] }
\end{aligned}
$$

where $C_{\mathrm{m}, \mathrm{T}}^{\mathrm{Sat}}$ is the solute $\left(\mathrm{mol} \cdot \mathrm{L}^{-1}\right)$ solubility in the solvent mixtures at temperature $T ; \varphi_{1}$ and $\varphi_{2}$ are volume fractions of solvents 1 (NMP) and 2 (water) in the absence of solute; $C_{1, T}^{\mathrm{Sat}}$ and $C_{2, \mathrm{~T}}^{\text {Sat }}$ denote the $\mathrm{mol} \cdot \mathrm{L}^{-1}$ solubility of the solute in solvents 1 and 2 , respectively; and $J_{i}$ are constants of the model computed by a regression analysis. These constants represent differences in various solute-solvent and solvent-solvent interactions in the mixture.

Equation 1 was fitted to the experimental solubility data of each drug, and the back-calculated solubilities were used to calculate the accuracy of the fitness. The mean deviation (MD) was used to check the accuracy and was calculated using

$$
\mathrm{MD}=\frac{\sum\left\{\frac{\left|\left(C_{\mathrm{m}}^{\mathrm{Sat}}\right)_{\text {pred }}-\left(C_{\mathrm{m}}^{\mathrm{Sat}}\right)\right|}{\left(C_{\mathrm{m}}^{\mathrm{Sat}}\right)}\right\}}{N}
$$

where $N$ is the number of data points in each set.

\section{Result and Discussion}

Table 2 lists the experimental solubilities of CZP, DZP, LTG, and PB in NMP + water mixtures at 298.2 K. There were good agreements between the reported solubility of LTG in water ${ }^{24}\left(0.000664 \mathrm{~mol} \cdot \mathrm{L}^{-1}\right.$ at $\left.298.15 \mathrm{~K}\right)$ and DZP in water $^{25}(0.00014817$ at $295.15 \mathrm{~K}$ to $297.15 \mathrm{~K})$ from the
Table 2. Experimental Solubilities of CZP, DZP, LTG, and PB in NMP (1) + Water (2) Mixtures at 298.2 K, Density $\rho$ of the

\begin{tabular}{|c|c|c|c|}
\hline \multirow[b]{2}{*}{$\varphi_{1}$} & \multirow[b]{2}{*}{$\rho / \mathrm{g} \cdot \mathrm{cm}^{-3}$} & \multicolumn{2}{|c|}{$C_{\mathrm{m}}^{\mathrm{Sat}} / \mathrm{mol} \cdot \mathrm{L}^{-1}$} \\
\hline & & experimental & calculated \\
\hline & & CZP & \\
\hline 0.00 & 1.004 & 0.00010 & 0.00010 \\
\hline 0.10 & 1.012 & 0.00035 & 0.00028 \\
\hline 0.20 & 1.015 & 0.00075 & 0.00077 \\
\hline 0.30 & 1.025 & 0.00173 & 0.00202 \\
\hline 0.40 & 1.033 & 0.00448 & 0.00514 \\
\hline 0.50 & 1.040 & 0.01354 & 0.01261 \\
\hline 0.60 & 1.052 & 0.02813 & 0.02990 \\
\hline 0.70 & 1.060 & 0.07433 & 0.06846 \\
\hline 0.80 & 1.067 & 0.16433 & 0.15138 \\
\hline 0.90 & 1.087 & 0.38975 & 0.32326 \\
\hline \multirow[t]{2}{*}{1.00} & 1.100 & 0.66671 & 0.66671 \\
\hline & & DZP & \\
\hline 0.00 & 1.002 & 0.00015 & 0.00015 \\
\hline 0.10 & 1.010 & 0.00366 & 0.00219 \\
\hline 0.20 & 1.015 & 0.00737 & 0.00856 \\
\hline 0.30 & 1.025 & 0.01259 & 0.01598 \\
\hline 0.40 & 1.033 & 0.01938 & 0.02213 \\
\hline 0.50 & 1.044 & 0.03349 & 0.03112 \\
\hline 0.60 & 1.054 & 0.06420 & 0.05329 \\
\hline 0.70 & 1.060 & 0.13502 & 0.11709 \\
\hline 0.80 & 1.065 & 0.28493 & 0.30516 \\
\hline 0.90 & 1.087 & 0.59634 & 0.76559 \\
\hline \multirow[t]{2}{*}{1.00} & 1.117 & 1.31710 & 1.31710 \\
\hline & & LTG & \\
\hline 0.00 & 1.000 & 0.00073 & 0.00073 \\
\hline 0.10 & 1.010 & 0.00321 & 0.00288 \\
\hline 0.20 & 1.017 & 0.00732 & 0.00742 \\
\hline 0.30 & 1.027 & 0.01243 & 0.01387 \\
\hline 0.40 & 1.038 & 0.02013 & 0.02057 \\
\hline 0.50 & 1.044 & 0.02946 & 0.02619 \\
\hline 0.60 & 1.050 & 0.03061 & 0.03058 \\
\hline 0.70 & 1.056 & 0.03283 & 0.03455 \\
\hline 0.80 & 1.052 & 0.04023 & 0.03939 \\
\hline 0.90 & 1.048 & 0.04576 & 0.04669 \\
\hline \multirow[t]{2}{*}{1.00} & 1.040 & 0.05853 & 0.05853 \\
\hline & & PB & \\
\hline 0.00 & 1.004 & 0.00533 & 0.00533 \\
\hline 0.10 & 1.008 & 0.11851 & 0.07184 \\
\hline 0.20 & 1.019 & 0.20182 & 0.26435 \\
\hline 0.30 & 1.033 & 0.38069 & 0.46778 \\
\hline 0.40 & 1.046 & 0.58587 & 0.60939 \\
\hline 0.50 & 1.069 & 0.94975 & 0.77761 \\
\hline 0.60 & 1.112 & 1.31473 & 1.12400 \\
\hline 0.70 & 1.137 & 1.64962 & 1.84987 \\
\hline 0.80 & 1.144 & 2.73952 & 3.02833 \\
\hline 0.90 & 1.175 & 3.88533 & 3.74434 \\
\hline 1.00 & 1.169 & 2.30766 & 2.30766 \\
\hline
\end{tabular}
Saturated Solutions, and the Back-Calculated Solubilities Using Equation 1

literature and the measured solubilities of LTG in water $\left(0.000729 \mathrm{~mol} \cdot \mathrm{L}^{-1}\right.$ at $\left.298.2 \mathrm{~K}\right)$ and DZP in water $(0.0001517$ at $298.2 \mathrm{~K}$ ) in this work. The computed solubilities of these drugs were compared via the fitness of eq 1 to experimental data and also the density of the saturated solutions. The solubilities of CZP, DZP, and LTG increased with the addition of NMP, and the maximum values are in neat NMP. The solubility of PB increased with the addition of NMP, reached the maximum value, and then decreased with a further increase in NMP concentration. Figures 1 and 2 illustrate the solubility profile of drugs in various volume fractions of NMP in the binary mixtures and the backcalculated solubilities by eq 1 . The calculated solubilities were compared with the corresponding experimental data, and MD values were computed and listed in Table 3 along with the model constants. The results show that the Jouyban-Acree model calculates the solubility of drugs in 


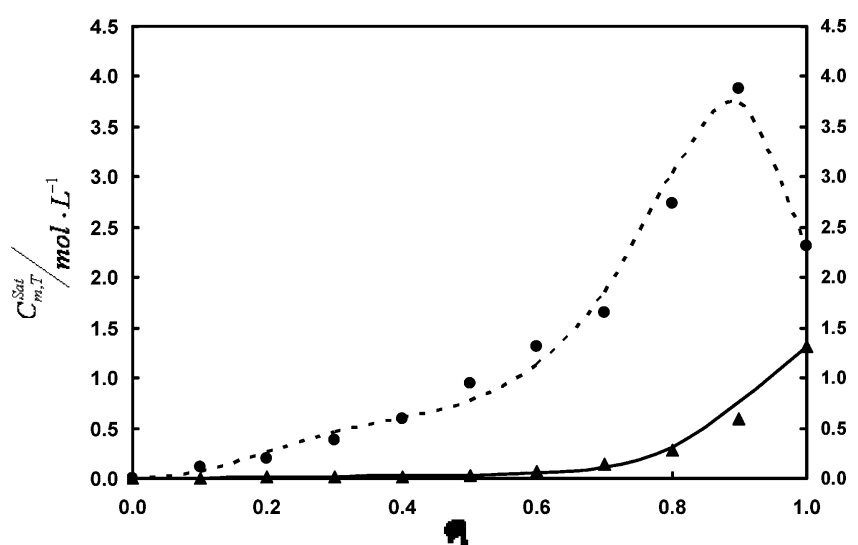

Figure 1. Experimental solubilities of diazepam and phenobarbital $\left(C_{\mathrm{m}, \mathrm{T}}^{\mathrm{Sat}} /\right.$ $\left.\mathrm{mol} \cdot \mathrm{L}^{-1}\right)$ at various volume fractions of $N$-methyl-2-pyrrolidone $\left(\varphi_{1}\right)$ in binary solvent mixtures: $\bullet$, phenobarbital; $\mathbf{\Delta}$, diazepam and the backcalculated solubilities using eq 1: - --, phenobarbital; -, diazepam.

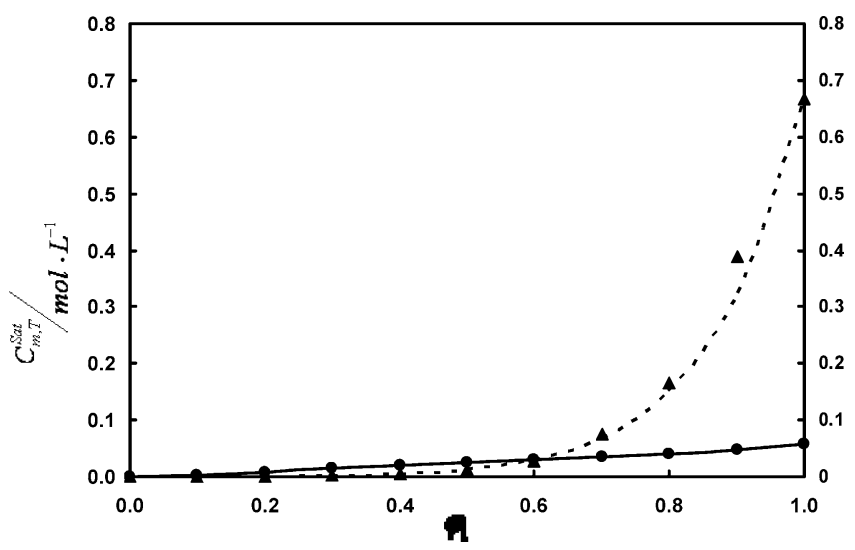

Figure 2. Experimental solubilities of clonazepam and lamotrigine $\left(C_{\mathrm{m}, \mathrm{T}}^{\mathrm{Sat}} /\right.$ $\left.\mathrm{mol} \cdot \mathrm{L}^{-1}\right)$ at various volume fractions of $N$-methyl-2-pyrrolidone $\left(\varphi_{1}\right)$ in binary solvent mixtures: $\boldsymbol{\bullet}$, lamotrigine; $\boldsymbol{\Lambda}$, clonazepam and the backcalculated solubilities using eq 1: -, lamotrigine; - - -, clonazepam.

Table 3. Numerical Values of the Model Constants and the Mean Deviation (MD) for the Back-Calculated Solubilities of CZP, DZP, LTG, and PB in NMP + Water Mixtures at 298.2 K Using Equation 1

\begin{tabular}{lcllr}
\hline drug & $J_{0}$ & \multicolumn{1}{c}{$J_{1}$} & \multicolumn{1}{c}{$J_{2}$} & $100 \cdot \mathrm{MD}$ \\
\hline CZP & 225.298 & - & - & 9.0 \\
DZP & 411.662 & -1264.200 & 1758.640 & 15.5 \\
LTG & 718.924 & -648.150 & 164.157 & 4.2 \\
PB & 1008.716 & -811.896 & 1891.487 & 14.2 \\
& \multicolumn{2}{c}{ Overall $100 \cdot \mathrm{MD}$} & & 10.7
\end{tabular}

NMP + water to within $10.7 \%$ error. More experimental solubility data sets are needed to present a generally trained version of the model for predicting the solubility of other drugs in NMP + water mixtures.

\section{Acknowledgment}

We thank Sobhan, Arastoo, and Pars Daru pharmaceutical companies for supplying the drug powders.

\section{Literature Cited}

(1) Alsenz, J.; Kansy, M. High Throughput Solubility Measurement in Drug Discovery and Development. Adv. Drug Delivery Rev. 2007, 59, 546-567.
(2) Strickley, R. G. Solubilizing Excipients in Oral and Injectable Formulations. Pharm. Res. 2004, 21, 201-230.

(3) Liu, R. Water-Insoluble Drug Formulation, 2nd ed.; CRC: Boca Raton, USA, 2008.

(4) Adjei, A.; Newburger, J.; Martin, A. Extended Hildebrand Approach. Solubility of Caffeine in Dioxane-Water Mixtures. J. Pharm. Sci. 1980, 69, 659-661.

(5) Li, A.; Yalkowsky, S. H. Predicting Cosolvency. 1. Solubility Ratio and Solute $\log \mathrm{K}_{\mathrm{ow}}$. Ind. Eng. Chem. Res. 1998, 37, 4470-4475.

(6) Williams, N. A.; Amidon, G. L. Excess Free Energy Approach to the Estimation of Solubility in Mixed Solvent System. II. Ethanol-Water Mixtures. J. Pharm. Sci. 1984, 73, 14-18.

(7) Ochsner, A. B.; Belloto, R. J., Jr.; Sokoloski, T. D. Prediction of Xanthine Solubilities Using Statistical Techniques. J. Pharm. Sci. 1985, 74, 132-135.

(8) Acree, W. E., Jr. Mathematical Representation of Thermodynamic Properties. Part II. Derivation of the Combined Nearly Ideal Binary Solvent (NIBS)/Redlich-Kister Mathematical Representation from a Two-Body and Three-Body Interactional Mixing Model. Thermochim. Acta 1992, 198, 71-79.

(9) Khossravi, D.; Connors, K. A. Solvent Effect on Chemical Processes. I: Solubility of Aromatic and Heterocyclic Compounds in Binary Aqueous-Organic Solvents. J. Pharm. Sci. 1992, 81, 371-379.

(10) Barzegar-Jalali, M.; Jouyban-Gharamaleki, A. A General Model from Theoretical Cosolvency Models. Int. J. Pharm. 1997, 152, 247-250.

(11) Jouyban-Gharamaleki, A. The Modified Wilson Model and Predicting Drug Solubility in Water-Cosolvent Mixtures. Chem. Pharm. Bull. 1998, 46, 1058-1061.

(12) Machatha, S. G.; Bustamante, P.; Yalkowsky, S. H. Deviation from Linearity of Drug Solubility in Ethanol/Water Mixtures. Int. J. Pharm. 2004, 283, 83-88.

(13) Jouyban, A. Review of the Cosolvency Models for Predicting Solubility of Drugs in Water-Cosolvent Mixtures. J. Pharm. Pharm. Sci. 2008, $11,32-58$.

(14) Avdeef, A. Solubility of Sparingly-Soluble Ionizable Drugs. Adv. Drug Delivery Rev. 2007, 59, 568-590.

(15) Xu, W. L.; Mao, F.; Zhao, H. K.; Wang, Y. Q.; Wang, J. Solubility of Anthracene in N, N-Dimethyformamide, N, N-Dimethylacetamide, and N-Methyl-2-Pyrrolidone. J. Chem. Eng. Data 2007, 52, 553-554.

(16) Wang, Q.; Xu, H.; Li, X. Solubilities of 4-Carboxybenzaldehyde and 1,4-Benzenedicarboxylic Acid in N-Methyl-2-Pyrrolidone in the Temperature Range from (343.2 to 468.2 K. J. Chem. Eng. Data 2005, $50,243-245$.

(17) Zhang, H.; Wang, J.; Chen, Y.; Zhang, M. Solubility of Sodium Cefotaxime in Different Solvents. J. Chem. Eng. Data 2007, 52, 982985.

(18) Li, Q. S.; Yi, Z. M.; Su, M. G.; Wang, S.; Wu, X. H. Solubility of Dioxopromethazine Hydrochloride in Different Solvents. J. Chem. Eng. Data 2008, 53, 301-302.

(19) Li, D. Q.; Liu, D. Z.; Wang, F. A. Solubilities of Terephthalaldehydic, p-Toluic, Benzoic, Terephthalic, and Isophthalic Acids in N-methyl2-pyrrolidone from 295.65 to 371.35 K. J. Chem. Eng. Data 2001, 46, 172-173.

(20) Sanghvi, R.; Narazaki, R.; Machatha, S. G.; Yalkowsky, S. H. Solubility Improvement of Drugs Using N-Methyl Pyrrolidone. AAPS PharmSciTech 2008, 9, 366-376.

(21) Shayanfar, A.; Fakhree, M. A. A.; Acree, W. E.; Jouyban, A. Solubility of Lamotrigine, Diazepam, and Clonazepam in Ethanol + Water Mixtures at 298.15 K. J. Chem. Eng. Data, 2009, 54, 1107-1109.

(22) Shayanfar, A.; Acree, W. E.; Jouyban, A. Solubility of Lamotrigine, Diazepam, Clonazepam and Phenobarbital in Propylene Glycol + Water Mixtures at 298.15 K. J. Chem. Eng. Data 2009, 54, 11531157.

(23) Higuchi, T.; Connors, K. A. Phase-Solubility Techniques. Adv. Anal. Chem. Instrum. 1965, 4, 117-212.

(24) Moffat, A. C. Clarke's Analysis of Drug and Poisons; Pharmaceutical Press: London, 2004.

(25) Loftsson, T.; Hreinsdoìttir, D. Determination of Aqueous Solubility by Heating and Equilibration: A Technical Note. AAPS PharmSciTech 2006, 7, e1-e4.

Received for review January 6, 2009. Accepted May 19, 2009. The financial support under grant No. 87-45 of the Drug Applied Research Center is gratefully acknowledged.

JE9000153 